\title{
Understanding the Pathogenesis of Slow-Transit Constipation: One Step Forward
}

\author{
Hanlin L. Wang ${ }^{1}$
}

Published online: 19 June 2015

(c) Springer Science+Business Media New York 2015

Chronic constipation is a common clinical complaint, particularly in persons aged $\geq 65$ or $<15$ years. According to the national ambulatory medical care survey data collected in 2004, it is the second most common ambulatory care diagnosis after gastroesophageal reflux disease in the United States, with 5.4 million prescriptions filled in addition to a very large number of nonprescription medications purchased by constipated patients [1]. Medical management of constipation may include the intake of bulking or wetting agents, or osmotic or stimulant laxatives, or undergoing behavioral modification, biofeedback therapy, sacral nerve stimulation, or even colonic pacing. Patients refractory to medical treatment may eventually require surgical interventions with partial or total colectomy [2]. A variety of structural, mechanical, metabolic, or functional causes can lead to chronic constipation [3], including slow-transit constipation.

Slow-transit constipation is a motility disorder characterized by markedly increased total bowel transit time when measured with radioactive markers, normal radiologically assessed bowel diameter, and no evidence of megacolon or aganglionosis. Since there are no diagnostic histologic features that can help determine its underlying etiology, the condition is frustrating not only to patients, gastroenterologists, and colorectal surgeons, but also to pathologists. Even with colectomy specimens, histologic examination can be completely normal or show only

Hanlin L. Wang

hanlinwang@mednet.ucla.edu

1 Department of Pathology and Laboratory Medicine, David Geffen School of Medicine, University of California at Los Angeles, 10833 Le Conte Avenue, Los Angeles, CA 90095, USA secondary nonspecific changes as a result of chronic constipation or its treatment effects [4]. Common abnormalities may include melanosis coli, cryptitis, crypt abscess, mucosal prolapse, and pneumatosis coli. Typically, no appreciable morphologic abnormalities are observed in the nerve plexuses, ganglion cells, and muscle fibers.

The pathogenesis of slow-transit constipation remains largely unknown. Earlier investigations have focused on quantitative alternations of the interstitial cells of Cajal (ICC), the pacemaker of the gastrointestinal tract. Using antibodies against c-kit (CD117) or DOG1 (discovered on GIST-1, also known as Ano1 and TMEM16A), a complete absence or significant reduction in the number of ICC in colon specimens resected from patients with slow-transit constipation in comparison with normal controls was reported [5, 6]. Wang et al. [7] even suggested a value of 7 ICCs/high power field in the inner circular muscle layer as a threshold value in order to confirm the diagnosis of slowtransit constipation. Yet, this measurement is difficult for pathologists to report due to the technical challenges of counting ICC density in clinical specimens, unless the cells are completely absent. The normal ICC count may also vary depending on detecting methods and the age of patients [8]. Furthermore, one study reported no relationship between ICC numbers and slow-transit constipation [9]. Only one study described decreased expression of $c$-kit mRNA and protein in colon specimens from patients with slow-transit constipation in comparison with controls [10]. However, $c$-kit gene mutations appear to be an infrequent event in this group of patients: in a study of 23 patients, only one case showed a missense mutation, although two polymorphism sites in the intron region were also detected, which may affect exon-intron splicing [11].

Many other pathophysiological abnormalities in the diseased colon may be associated with slow-transit 
constipation. Examples include degeneration of the myenteric plexus ganglia, reduced cholinergic and enhanced adrenergic responses, an attenuated gastrocolic reflex, rectosigmoid dyssynergia, aberrant expression of smoothelin protein, and abnormalities of the enteric neurotransmitters such as substance $P$, vasoactive intestinal peptide, pancreatic polypeptide, peptide YY, neuropeptide Y, cholecystokinin, nitric oxide, and ovarian and adrenal steroid hormones $[4,12]$. How these numerous abnormalities contribute to the pathogenesis of slow-transit constipation remain to be further elucidated.

In this issue of Digestive Diseases and Sciences, Liu et al. [13] took a different approach to investigate the pathogenesis of slow-transit constipation. The authors first established that the number of macrophages was significantly increased, correlated with down-regulation of microRNA-128 expression, in 20 of 25 (80\%) colon specimens resected from patients with slow-transit constipation in comparison with those from patients without a history of constipation. The authors further provided evidence that microRNA-128 might directly target p38 $\alpha$ (a protein kinase) in intestinal epithelial cells to regulate the expression of macrophage colony-stimulating factor (M-CSF). The authors hypothesize that these mechanisms increase the recruitment of macrophages into the colon, which activate an immune reaction that contributes to the pathogenesis of slow-transit constipation. Interestingly, the authors also observed a reduction in the number of ICCs in diseased colon, further supporting published data.

As the first study implicating macrophages and microRNAs in the pathogenesis of slow-transit constipation, the study draws on considerable data linking macrophage and microRNA functions to a specific bowel disease. It is well known that macrophages are central players in innate and adaptive defense mechanisms that are essential for the maintenance of tissue homeostasis and the control of inflammation and immunity as integral components of their host tissues [14]. On the other hand, microRNAs, the noncoding regulatory RNA composed of 21-23 nucleotides, are capable of regulating thousands of genes involved in diverse biological processes such as cell differentiation and development [15]. As a result, macrophages and microRNAs are implicated in the pathogenesis of numerous human diseases such as cancers, autoimmune diseases, and developmental disorders among many others, becoming prime targets of therapeutic interventions. As an example, microRNA-128, highly expressed in the central nervous system, is important for neural stem cell self-renewal and differentiation and is also capable of regulating the differentiation of rat bone mesenchymal stem cells into neuron-like cells by Wnt signaling [16]. It remains to be investigated whether decreased microRNA-128 expression in slow-transit constipation can regulate the proliferation and/or differentiation of enteric neural cells and ICCs.

Although patients with slow-transit constipation may have a higher number of mast cells in diseased colon specimens in comparison with controls [17], depletion of mast cells in $W / W^{v}$ mice that lack ICCs generates an inflammatory environment in the muscularis externa that suppresses smooth muscle contractility. Depletion of mast cells in this mouse model also suppresses the expression of choline acetyltransferase (ChAT) and neural nitric oxide synthase (nNOS) in myenteric neurons, which may impair neuronal function. These observations suggest that mast cells are implicated in the pathogenesis of gastrointestinal motility disorders independent of the loss of ICC [18]. It would be interesting to investigate the roles of macrophages in this mouse model to explore their relationship with ICCs.

In summary, the observations that macrophages and microRNA are involved in the pathogenesis of slow-transit constipation are scientifically insightful. They are also potentially important to the diagnosis and treatment of this frustrating disease if the findings can be reproduced in a larger number of cases.

\section{References}

1. Everhart JE, Ruhl CE. Burden of digestive diseases in the United States part II: lower gastrointestinal diseases. Gastroenterology. 2009;136:741-754.

2. Bassotti G, Blandizzi C. Understanding and treating refractory constipation. World J Gastrointest Pharmacol Ther. 2014;5:77-85.

3. Ouyang A, Locke GR 3rd. Overview of neurogastroenterologygastrointestinal motility and functional GI disorders: classification, prevalence, and epidemiology. Gastroenterol Clin North Am. 2007;36:485-498.

4. Chan OT, Chiles L, Levy M, et al. Smoothelin expression in the gastrointestinal tract: implication in colonic inertia. Appl Immunohistochem Mol Morphol. 2013;21:452-459.

5. He CL, Burgart L, Wang L, et al. Decreased interstitial cell of Cajal volume in patients with slow transit constipation. Gastroenterology. 2000;118:14-21.

6. Geramizadeh B, Hayati K, Rahsaz M, et al. Assessing the interstitial cells of Cajal, cells of enteric nervous system and neurotransmitters in slow transit constipation, using immunohistochemistry for CD117, PGP9.5 and serotonin. Hepatogastroenterology. 2009;56:1670-1674.

7. Wang LM, McNally M, Hyland J, et al. Assessing interstitial cells of Cajal in slow transit constipation using CD117 is a useful diagnostic test. Am J Surg Pathol. 2008;32:980-985.

8. Knowles $\mathrm{CH}$, Farrugia G. Gastrointestinal neuromuscular pathology in chronic constipation. Best Pract Res Clin Gastroenterol. 2011;25:43-57.

9. Toman J, Turina M, Ray M, et al. Slow transit colon constipation is not related to the number of interstitial cells of Cajal. Int $J$ Colorectal Dis. 2006;21:527-532.

10. Tong WD, Liu BH, Zhang LY, et al. Expression of c-kit messenger ribonucleic acid and c-kit protein in sigmoid colon of 
patients with slow transit constipation. Int $J$ Colorectal Dis. 2005;20:363-367.

11. Tong WD, Liu BH, Zhang LY, et al. Analysis of the $c$-kit gene in patients with slow transit constipation. Gut. 2006;55:1207-1208.

12. Andromanakos NP, Pinis SI, Kostakis AI. Chronic severe constipation: current pathophysiological aspects, new diagnostic approaches, and therapeutic options. Eur J Gastroenterol Hepatol. 2015;27:204-214.

13. Liu W, Zhang Q, Li S, et al. The relationship between colonic macrophages and microRNA-128 in the pathogenesis of slow transit constipation. Dig Dis Sci. (Epub ahead of print). doi:10. 1007/s10620-015-3612-1.

14. Varol C, Mildner A, Jung S. Macrophages: development and tissue specialization. Annu Rev Immunol. 2015;33:643-675.
15. Song JL, Nigam $\mathrm{P}$, Tektas SS, et al. MicroRNA regulation of Wnt signaling pathways in development and disease. Cell Signal. 2015;27:1380-1391.

16. Wu R, Tang Y, Zang W, et al. MicroRNA-128 regulates the differentiation of rat bone mesenchymal stem cells into neuron-like cells by Wnt signaling. Mol Cell Biochem. 2014;387:151-158.

17. Bassotti G, Villanacci V, Nascimbeni R, et al. Colonic mast cells in controls and slow transit constipation patients. Aliment Pharmacol Ther. 2011;34:92-99.

18. Winston JH, Chen J, Shi XZ, et al. Inflammation induced by mast cell deficiency rather than the loss of interstitial cells of Cajal causes smooth muscle dysfunction in $W / W^{v}$ mice. Front Physiol. 2014;5:22. 